ARTIGO ORIGINAL

\title{
Crescimento e produção em povoamentos de Pinus taeda L. danificados por macacos-prego
}

\author{
Growth and production in Pinus taeda L. stands damaged by capuchin monkeys
}

\author{
Franciéle Maria de Souza Retslaff ${ }^{1}$ (D), Afonso Figueiredo Filho ${ }^{1}$ (D) Fabiane Aparecida de Souza Retslaff ${ }^{1}$ (D), \\ José Robyson Aggio Molinari ${ }^{1}$ (D) \\ ${ }^{1}$ Universidade Estadual do Centro-Oeste - UNICENTRO, Irati, PR, Brasil
}

Como citar: Retslaff, F. M. S., Figueiredo Filho, A., Retslaff, F. A. S., \& Molinari, J. R. A. (2020). Crescimento e produção em povoamentos de Pinus taeda L. danificados por macacos-prego. Scientia Forestalis, $48(125)$, e3237. https://doi.org/10.18671/scifor.v48n125.05

\begin{abstract}
Resumo
O objetivo dessa pesquisa foi avaliar as perdas de crescimento de árvores de Pinus taeda danificadas por macacos-prego. Para reconstruir o crescimento passado das variáveis diâmetro, área transversal, altura e volume, nas várias idades, foi aplicada a técnica de análise de tronco completa (ANATRO). O modelo de Chapman-Richards foi utilizado para descrever as curvas de produção e as curvas de incremento corrente anual (ICA) e incremento médio anual (IMA). O teste de igualdade de parâmetros e identidade de modelos de regressão não-linear foi aplicado nas curvas de produção a fim de verificar a possibilidade de agrupar ou não os dados de árvores não danificadas e danificadas com dano anelar e dano janelar. Os resultados gerados pela ANATRO indicaram que os primeiros danos surgiram entre 5-6 anos de idade, porém a maioria ocorreu entre 7-8 anos. A altura foi a variável mais afetada com os danos de macacos-prego, reduzindo em, aproximadamente, $50 \%$ seu potencial de crescimento para danos do tipo anelamento e, por consequência, afetando o volume. As árvores janeladas apresentaram volume médio individual superior ao das árvores não danificadas e o teste de Regazzi (2003) apontou diferença entre as curvas de crescimento para as variáveis diâmetro (DAP), altura (h) e volume (v), não sendo possível o uso da mesma equação para os três grupos de árvores avaliados (aneladas, janeladas e não danificadas).
\end{abstract}

Palavras-chave: Sapajus nigritus; Incremento; Danos florestais.

\begin{abstract}
The objective of this research was to evaluate the growth losses of trees of Pinus taeda damaged by capuchin monkeys. To reconstruct the past growth of variables diameter, individual basal area, height and volume, at various ages, the technique of complete stem analysis (ANATRO) was applied. The Chapman-Richards model was used to describe the production curves and the current annual increment (CAI) and mean annual increment (MAl) curves. The Regazzi test (2003) was applied to the production curves to verify the possibility of grouping or not the data of non-damaged and damaged trees, with ring damage and window damage. The results generated by ANATRO indicated that the first damage occurred between 5-6 years of age, but most occurred between 7-8 years old. Height was the variable most affected by the damage of the Capuchin monkeys, reducing its growth potential by approximately 50\% for ringing damages and, consequently, affecting volume. The windowed trees presented a higher individual medium volume compared to not damaged group. This happened due to the cicatrization process of the fissure caused by the monkeys as well as due the excentricity of the transversal section where the damage occurred. The Regazzi test (2003) showed a difference between the growth curves for the variables diameter (dbh), height (h) and volume (v), and it was not possible to use the same equation for the three groups of trees evaluated (ringed, windowed and non-damaged).
\end{abstract}

Keywords: Sapajus nigritus; Increment; Forest damage.

Fonte de financiamento: Nenhuma.

Conflito de interesse: Nada a declarar.

Autor correspondente: francieleretslaff@yahoo.com.br

Recebido: 4 janeiro 2019.

Aceito: 7 maio 2019.

Editor: Francides Gomes da Silva Júnior.

(c) (i) Este é um artigo publicado em acesso aberto (Open Access) sob a licença Creative Commons Attribution, que permite uso, distribuição e reprodução cc. em qualquer meio, sem restrições desde que o trabalho original seja corretamente citado. 


\section{INTRODUÇÃO}

Os primeiros danos causados por macacos-prego em plantios de Pinus spp. no sul do Brasil foram registrados na década de 1950, entretanto, foi na década de 1990 que os danos aos povoamentos aumentaram de intensidade (Rocha, 2000). Nas últimas décadas os danos estão mais frequentes, provavelmente em função do aumento da área plantada e do monitoramento florestal (Mikich et al., 2015).

Os plantios florestais de Pinus spp. ocupam 1,6 milhão de hectares e concentram-se principalmente nos estados do Paraná (42\%), Santa Catarina (34\%) e Rio Grande do Sul (12\%) (Indústria Brasileira de Árvores, 2017), caracterizando uma distribuição geográfica bastante similar ao do macaco-prego (Sapajus nigritus), que vai do sul de Minas Gerais ao norte do Rio Grande do Sul, e também em parte da Argentina (Alfaro et al., 2012). Para os pesquisadores Mikich et al. (2015), os danos dos macacos-prego aos plantios florestais são críticos nas regiões em que a floresta nativa foi ou é explorada/suprimida, pois isso reduz a abundância de espécies que servem de alimento para o macaco-prego.

O descascamento do fuste pelos macacos-prego pode levar a morte da árvore e comprometer o crescimento. Ainda são poucos os trabalhos sobre como os danos dos macacos-prego afetam o crescimento das árvores de Pinus taeda, dentre os quais está o Carvalho (2007). Este Autor buscou levantar informações sobre as quantidades de árvores de Pinus spp. que foram danificadas por macacos-prego, identificando os danos ocorridos e se existiam diferenças estatisticamente significativas entre os diâmetros (diâmetro a 1,3 m do solo - DAP) das árvores danificadas e não danificadas, em plantios da espécie nos municípios de Ventania e Jaguariaíva, estado do Paraná.

Liebsch et al. (2015) descreveram os efeitos dos danos causados pelo macaco-prego sobre o incremento em diâmetro e volume em plantios de $P$. taeda. Os autores avaliaram o incremento em diâmetro a altura do peito (DAP) a partir do momento do dano até a idade final, e puderam observar que nas árvores com danos do tipo janela (como os autores classificaram), a perda em crescimento foi mais acentuada em árvores jovens, variando de 3,5 a $0,7 \%$ aos 5,5 anos e 10 anos de idade, respectivamente. Já para as árvores que sofreram ferimentos do tipo anelamento, na primeira avaliação (5,5 anos) a perda foi de $25,7 \%$, caindo gradativamente chegando a $4,2 \%$ aos 10 anos.

Em termos volumétricos, Liebsch et al. (2015) compararam indivíduos danificados com indivíduos sem danos e observaram que o dano do tipo janela ocasionou uma perda de $7,0 \%$ aos 5,5 anos, atingindo $1,4 \%$ aos 10 anos de idade. Para o dano tipo anelamento o incremento em volume sofreu uma redução de $44,9 \%$ quando os danos foram ocasionados aos 5,5 anos, sendo que esse valor caiu gradativamente nos anos seguintes, chegando a $8,2 \%$ aos 10 anos.

Oliveira et al. (2015), utilizando-se dos dados do trabalho de Liebsch et al. (2015), apresentaram um procedimento para calibrar o software SisPinus visando projetar o crescimento e a produção de povoamentos danificados por macaco-prego, em função de diferentes níveis de danos e idades de ocorrência dos mesmos.

Ainda, pode-se citar o trabalho de Pavelski (2016) que utilizou o software SisPinus para simular o crescimento dos talhões e o crescimento corrigido dos mesmos, para determinar perdas de volume. Esse Autor utilizou as planilhas desenvolvidas por Oliveira et al. (2015), chegando a valores de perdas em volume nos talhões danificados que variaram entre $12,6 \mathrm{~m}^{3} \cdot$ ha $^{-1}(2,30 \%)$ a $47,3 \mathrm{~m}^{3} \cdot$ ha $^{-1}(8,96 \%)$ no final de um ciclo de 18 anos.

Apesar da relevância do tema para o setor florestal, são poucas as informações sobre como os danos provocados por macacos-prego afetam o crescimento das árvores. Muitos produtores avaliam suas perdas contabilizando apenas árvores com $100 \%$ de perda (árvores mortas) sem considerar a possível perda de incremento anual. Dados mais acurados, por exemplo, se existe a perda de incremento anual em árvores que foram danificadas, mas não morreram pelo dano, podem gerar uma visão mais realista da situação (Jungles \& Vidolin, 2009).

Diante do exposto e considerando a importância dos plantios de Pinus taeda L. para o setor florestal do sul do Brasil, a presente pesquisa buscou avaliar o crescimento passado de árvores danificadas e não danificadas por macacos-prego e avaliar o efeito dos danos dos 
macacos-prego no crescimento em diâmetro, altura, área transversal e volume das árvores. Buscou-se ainda, identificar por meio da técnica de análise de tronco completa à idade em que ocorreram os primeiros danos ocasionados por macacos-prego nas árvores de Pinus taeda.

\section{MATERIAL E MÉTODOS}

\section{Área de estudo}

Os dados foram coletados em um povoamento de Pinus taeda, localizado na região Centro-Sul do estado do Paraná, Brasil. Foram amostrados dois talhões estabelecidos em 1998 com espaçamento inicial de 3,0 × 2,5 m, os quais receberam os mesmos tratamentos silviculturais, além de pertencerem ao mesmo sítio. No primeiro talhão, com área de 13,81 ha, foram coletadas informações de árvores que não foram danificadas pelo macaco-prego. No segundo talhão, com área de 14,12 ha, as árvores foram severamente danificadas por macacos-prego, sendo coletado deste talhão dados de árvores danificadas. Foram instaladas previamente duas parcelas, distribuídas aleatoriamente nos talhões, sendo uma parcela com área de 01 hectare no talhão não danificado e uma parcela de 01 hectare no talhão danificado por macacos-prego. Nas parcelas instaladas nos dois talhões (não danificado e danificado) foram medidos todos os diâmetros à altura do peito (DAP) e todas as alturas totais.

\section{Análise de tronco completa (ANATRO)}

Para recuperar o crescimento passado, identificar a provável idade de ocorrência dos danos pelo macaco-prego e avaliar a interferência dos danos no crescimento foi utilizada a técnica de análise do tronco completa (ANATRO). A partir do inventário florestal, os dados foram agrupados em classes diamétricas com amplitude de $3 \mathrm{~cm}$. A fórmula de Sturges foi empregada para definir o número de classes de diâmetro, para as árvores danificadas e não danificadas pelo macaco-prego. Foram coletados discos de 40 árvores com danos e 40 sem danos do macaco-prego, distribuídas em todas as classes diamétricas. As árvores danificadas foram classificadas de acordo com o tipo de dano anelamento ou janelamento.

Os discos para a análise de tronco foram coletados nas alturas absolutas de 0,10 e 1,30 m e nas alturas relativas de 15, 30, 45, 60, 75 e 90\% da altura total da árvore, totalizando 663 discos. Para as árvores aneladas foi considerada a altura até o ponto onde ocorreu o anelamento. Foram coletados 8 discos em cada árvore não danificada, 8 discos em cada árvore janelada e 9 discos em cada árvore anelada, dos quais, 8 do fuste principal e um da base de maior brotação, que surge após a quebra da ponteira da árvore pelos macacos-prego (Figura 1).

Os discos foram numerados, secos e lixados para obter uma melhor visualização dos anéis na face utilizada para as medições. Então, foram marcados 4 raios para as medições dos anéis de crescimento (Figura 1).
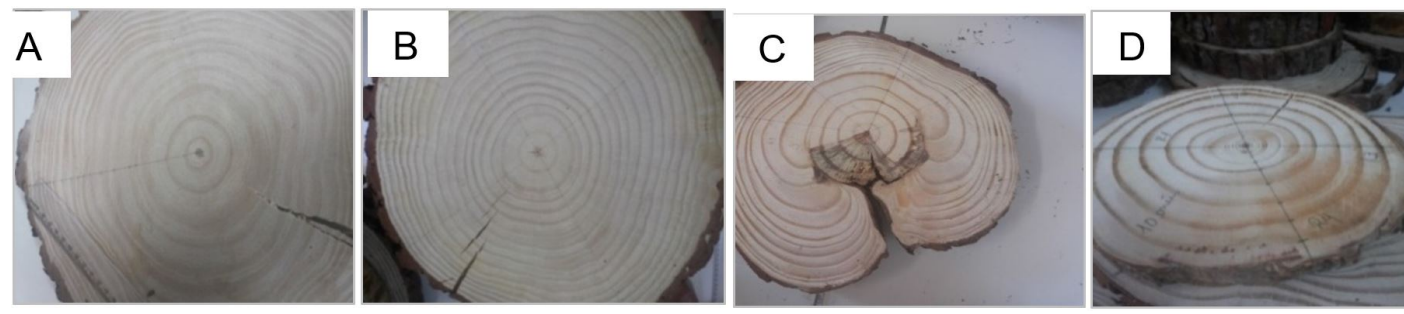

Figura 1. Preparo do material para medição dos anéis: marcação dos raios e identificação dos anéis nos discos (A, B). Marcação dos discos danificados por macacos-prego (C, D).

Para a contagem e medição dos anéis foi utilizado o medidor de anéis de crescimento LINTAB 6 do Laboratório de Manejo Florestal da UNICENTRO, Campus de Irati. O processamento dos dados foi realizado com o suplemento FlorExcel ${ }$, o qual gera 
informações do crescimento anual em diâmetro, área transversal, altura total e volume. Todas essas informações foram obtidas para a idade atual (18 anos) e para todas as idades passadas da árvore.

A análise de tronco completa possibilita a medição do diâmetro com casca apenas para idade de corte ou idade atual. Nas idades anteriores têm-se apenas diâmetros sem casca e, como consequência, todas as variáveis geradas (diâmetro, área transversal e volume) são sem casca (Wolff II, 2012). Todavia, os sortimentos do volume utilizam bitolas com casca e, assim, foi empregada a Equação 1 ajustada por Wolff II (2012) para estimar os diâmetros com casca ao longo do fuste nas idades passadas para cada árvore amostrada. A equação de Wolff II (2012) apresentou estatísticas de ajuste e precisão, respectivamente, de $\mathrm{R}^{2}=0,9983$ e $\mathrm{S}_{\mathrm{yx}}=3,14 \%$.

$d_{c / c}=0,024184+1,0662557 \cdot d_{s / c}$

Em que: $d_{c / c}=$ diâmetro com casca ao longo do fuste $(\mathrm{cm}) ; d_{s / c}=$ diâmetro sem casca ao longo do fuste $(\mathrm{cm})$.

\section{Modelagem do crescimento e produção}

Para descrever as curvas de crescimento e produção das variáveis estudadas (DAP, g, v, h) foi utilizado o modelo biológico de Chapman-Richards (Equação 2). Os dados para o ajuste foram

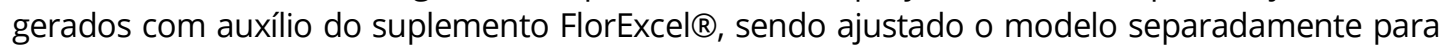
cada grupo de árvores (não danificada e danificadas por macacos-prego). Para as árvores danificadas foi realizado um ajuste para cada tipo de dano (janelamento e anelamento), a fim de comparar o crescimento médio entre os indivíduos que sofreram danos com os indivíduos que não sofreram danos por macacos-prego.

$$
y=A \cdot\left[1-e^{(-b \cdot I)}\right]^{c}
$$

Em que: $y$ = variável dependente ( $d$, altura, área transversal e volume); A, b e c = parâmetros a serem estimados do modelo em que $\mathrm{A}=$ valor assintótico; $\mathrm{b}=$ controla a taxa de crescimento e $c=$ parâmetro que controla o ponto de inflexão; $e=$ exponencial; $I$ = Idade (anos).

Os talhões tinham uma diferença de idade de aproximadamente 6 meses e, assim, as análises foram realizadas até os 18 anos para ambos os talhões, considerando-se os anéis a partir de 3 anos de idade, devido às distorções causadas pelas dificuldades de identificação e medição dos anéis nas primeiras idades. $O$ incremento corrente anual (ICA) e o incremento médio anual (IMA) foram obtidos com base nas Equações 3 e 4, utilizando os valores estimados pelo modelo de Chapman-Richards. Esses valores foram utilizados para a construção de curvas de incremento para cada uma das variáveis, com objetivo de identificar possíveis alterações no incremento de árvores danificadas por macacos-prego comparadas com as árvores não danificadas.

$I C A_{y}=y_{i+1}-y_{i}$

$I M A_{y}=\frac{y_{i}}{i}$

Em que: $I C A_{y}=$ incremento corrente anual da variável $y$ (DAP, altura, área transversal e volume); $y_{i+1}$ : variável na idade $i+1 ; i$ : idade (anos); $y_{i}$ : variável na idade i; IMA $y$ : incremento médio anual da variável $y$. 


\section{Comparações da produção das árvores danificadas e não danificadas pelo macaco-prego}

O teste de igualdade de parâmetros e identidade de modelos de regressão não-linear proposto por Regazzi (2003) foi usado para verificar se as curvas de produção geradas pelo ajuste de Chapman-Richards, que descrevem o crescimento médio das variáveis (DAP, g, h e v), eram estatisticamente iguais para os três grupos de árvores estudados (aneladas, janeladas e não danificadas). O teste indica se uma única equação pode ser usada para a estimativa da produção média de uma determinada variável para os três grupos de árvores estudados (não danificadas, aneladas e janeladas) ou se é necessária uma equação para cada grupo. Avalia ainda, se um subconjunto de parâmetros é igual para os três grupos estudados. Assim, foram testadas as seguintes hipóteses:

(a) $H_{0(1):}: A_{1}=A_{2}=A_{3} ; H_{1(1)}:$ nem todos os $A_{(i)}$ são iguais;

$H_{0(2)}: b_{1}=b_{2}=b_{3} ; H_{1(2)}$ : nem todos os $b_{(i)}$ são iguais;

$H_{0(3)}: c_{1}=c_{2}=c_{3} ; H_{1(3)}$ : nem todos os $c_{(i)}$ são iguais;

(b) $H_{0(4)}$ : $A_{1}=A_{2}=A_{3}$ e $b_{1}=b_{2}=b_{3}$ e $c_{1}=c_{2}=c_{3}$ as funções de crescimento são idênticas para árvores aneladas, janeladas e não danificadas, isto é, uma função comum pode ser usada como estimativa para os três conjuntos de dados (nesta hipótese testa se os coeficientes das três equações de crescimento são iguais para os três grupos aneladas, janeladas e não danificadas); $H_{1}(4)$ : pelo menos uma igualdade é uma desigualdade, ou seja, pelo menos um coeficiente é diferente.

Para testar as hipóteses $\mathrm{H}_{01}, \mathrm{H}_{02}$ e $\mathrm{H}_{03}$ foi necessário avaliar cada coeficiente separadamente. No ajuste fixou-se um a um e testou-se a hipótese de ser igual ou diferente em cada grupo de dados.

Para a realização do teste, as três equações de crescimento foram ajustadas com variáveis "dummy", onde um modelo completo (ômega) e um modelo reduzido (w) são ajustados.

O modelo completo (Equação 5), foi ajustado com os coeficientes diferentes para os três grupos de dados (não danificadas, janeladas e aneladas) e no modelo reduzido (Equação 6), foi imposta a restrição de que os coeficientes do modelo para os três grupos devem ser iguais, ou seja, uma única equação pode ser usada para os três grupos de dados.

Modelo completo:

$Y=d 1 *\left(A 1^{*}\left(1-\exp \left(-b 1^{*} x\right)\right)^{C 1}\right)+d 2 *\left(A 2 *(1-\exp (-b 2 * x))^{C 2}\right)+d 3^{*}\left(A 3 *(1-\exp (-b 3 * x))^{C 3}\right)$

Modelo reduzido:

$Y=d 1^{*}\left(A^{*}\left(1-\exp \left(-b^{*} x\right)\right)^{C}\right)+d 2^{*}\left(A^{*}\left(1-\exp \left(-b^{*} x\right)\right)^{C}\right)+d 3^{*}\left(A^{*}\left(1-\exp \left(-b^{*} x\right)\right)^{C}\right)$

Em que: $\mathrm{Y}=$ variável estimada (DAP, $\mathrm{g}, \mathrm{h}$ e v); $\mathrm{x}=$ idade; $\mathrm{d}=$ variável Dummy; A, b, c = coeficientes do modelo, as numerações 1, 2 e 3 referem-se aos grupos: 1 refere-se as árvores não danificadas, 2 às janeladas e 3 árvores aneladas.

A estatística do teste é apresentada a seguir (Equação 7):

$\chi_{\text {calculado }}^{2}=-n \ln \left(\frac{S Q R_{\Omega}}{S Q R_{w}}\right)$

Em que: $S Q R_{\Omega}=$ Soma de quadrados de resíduo do modelo completo $(\Omega) ; S Q R_{w}=$ soma de quadrados do modelo reduzido $(w) ; n=$ número total de observações. 
O teste apresenta a seguinte regra de decisão (Regazzi, 2003):

Se $\chi^{2}$ calculado $\geq \chi^{2}$ tabelado, rejeita-se $\mathrm{H}_{0}$. Caso contrário não se rejeita $\mathrm{H}_{0}$.

O valor tabelado foi obtido em função do nível de significancia $a=5 \%$ e do número de graus de liberdade $v\left(p_{\Omega}-p_{w}\right)$.

Em que: $v=$ número de graus de liberdade; $p_{\Omega}=$ número de coeficientes do modelo completo $p_{w}=$ número de coeficientes dos modelos reduzidos.

\section{RESULTADOS E DISCUSSÃO}

Os coeficientes e as estatísticas de ajuste e precisão para o modelo de Chapman-Richards obtido para cada variável estudada estão descritos na Tabela 1. Para a variável DAP, os valores dos coeficientes " $\mathrm{A}$ " do modelo ajustado, que representa o valor assintótico, apresentaram-se coerentes com a lógica biológica esperada para a espécie, variando de 35 a $40 \mathrm{~cm}$. Esperava-se valores maiores de assíntota, para árvores não danificadas, porém os resultados foram contrários. O diâmetro para as árvores aneladas nos primeiros anos apresentou-se superior aos diâmetros das árvores não danificadas e também superior aos diâmetros das árvores janeladas. Isso leva a crer que, no passado, essas árvores aneladas foram, até um determinado período, árvores dominantes e codominantes. Liebsch et al. (2015) descreveram em seu estudo intitulado "Descascamento de Pinus taeda por macacos-prego (Sapajus nigritus): tipos e intensidades de danos e seus impactos sobre o crescimento das árvores" que os macacos-prego atacam árvores em todas as classes de diâmetro, mas que têm preferência por árvores dominantes ou as mais vigorosas dos plantios, devido a maior concentração de seiva nessas árvores, da qual o macaco se alimenta. Desta forma, nos primeiros ataques os macacos-prego preferem as árvores dominantes e de diâmetros maiores, mas com o passar do tempo, se o ataque ocorre em grande intensidade, eles vão descascando também árvores mais finas.

O povoamento passou por 2 desbastes, no primeiro desbaste, segundo registros, foi buscado retirar as árvores que foram danificadas (as dominantes), porém nem todas foram retiradas mantendo-se no talhão árvores que apesar de danificadas possuíam potencial para obter produtos mais nobres no futuro.

As árvores que sofreram danos do tipo janelamento tiveram menor erro padrão de estimativa $(17,14 \%)$ para a estimativa da variável DAP.

Tabela 1. Parâmetros estimados do modelo de Chapman-Richards para as variáveis DAP com casca, altura total, área transversal com casca e volume total com casca e respectivas estatísticas de ajuste e precisão, para árvores aneladas, janeladas e árvores não danificadas de Pinus taeda.

\begin{tabular}{|c|c|c|c|c|c|}
\hline \multicolumn{6}{|c|}{ DAP com casca $(\mathrm{cm})$} \\
\hline & $A$ & B & $C$ & $\mathbf{R}^{2}$ aj. & $S_{y x}(\%)$ \\
\hline Aneladas & 36,01350 & 0,140550 & 1,617260 & 0,7518 & 20,22 \\
\hline Janeladas & 40,06740 & 0,110668 & 1,538580 & 0,8354 & 17,14 \\
\hline Não danificadas & 35,24700 & 0,151430 & 1,985650 & 0,8098 & 19,01 \\
\hline \multicolumn{6}{|c|}{ Altura total (m) } \\
\hline & $A$ & $B$ & C & $\mathbf{R}^{2}$ aj. & $S_{y x}(\%)$ \\
\hline Aneladas & 11,53120 & 0,483096 & 4,597660 & 0,6030 & 19,23 \\
\hline Janeladas & 24,42660 & 0,141331 & 1,900230 & 0,9154 & 12,07 \\
\hline Não danificadas & 32,62960 & 0,097023 & 1,738490 & 0,9430 & 10,77 \\
\hline \multicolumn{6}{|c|}{ Área transversal com casca $\left(\mathrm{m}^{2}\right)$} \\
\hline & $\boldsymbol{A}$ & B & C & $\mathbf{R}^{2}$ aj. & $S_{y x}(\%)$ \\
\hline Aneladas & 0,15025 & 0,087157 & 2,589600 & 0,6739 & 38,91 \\
\hline Janeladas & 0,279524 & 0,045138 & 2,014303 & 0,7221 & 37,72 \\
\hline Não danificadas & 0,12066 & 0,111410 & 2,987440 & 0,6938 & 40,51 \\
\hline \multicolumn{6}{|c|}{ Volume total com casca $\left(\mathrm{m}^{3}\right)$} \\
\hline & A & B & C & $\mathbf{R}^{2}$ aj. & $S_{y x}(\%)$ \\
\hline Aneladas & 1,49100 & 0,095982 & 3,472070 & 0,6344 & 51,94 \\
\hline Janeladas & 1,795180 & 0,114882 & 4,998330 & 0,7609 & 45,11 \\
\hline Não danificadas & 2,219920 & 0,087932 & 3,965960 & 0,7655 & 43,55 \\
\hline
\end{tabular}

Em que: $\mathrm{R}^{2}$ = coeficiente de determinação; $\mathrm{S}_{\mathrm{yx}} \%$ = erro padrão de estimativa; $b_{\text {is }}=$ parâmetros estimados. 
Pacheco (2013) utilizou o modelo biológico de Chapman-Richards para estimar o crescimento acumulado do DAP de $P$. taeda para um plantio com espaçamento de $3,0 \times 2,5$ m e obteve um coeficiente de determinação de 0,91 e erro padrão de $33,84 \%$.

Para o ajuste das alturas das árvores aneladas foi considerada a altura até o ponto de anelamento (hPA). Segundo Liebsch et al. (2018) essa altura é determinante para a produção de toras, sendo que acima desse dano o volume é perdido pela morte e seca da ponteira. Para a variável altura, o menor erro foi obtido para as árvores não-danificadas por macacos, com $\mathrm{S}_{\mathrm{yx}}$ de $10,77 \%$, seguido das árvores que sofreram danos do tipo janelamento com $\mathrm{S}_{\mathrm{yx}}$ de 12,07\%. Pacheco (2013) estimando o crescimento acumulado em altura obteve um erro padrão de estimativa de $20,91 \%$.

O valor assintótico estimado pelo modelo de Chapman-Richards " $\mathrm{A}$ " para altura das árvores não danificadas prevê que as árvores não danificadas podem atingir 32,62 m, enquanto nas janeladas, esse valor máximo foi estimado em $24,42 \mathrm{~m}$. Para as árvores que foram aneladas a altura assintótica estimada foi de 11,53 m, indicando que os danos causados pelos macacos-prego prejudicam, sobremaneira, o crescimento dessa variável. Para a estimativa do volume total com casca $\left(\mathrm{m}^{3}\right)$, o modelo apresentou erros elevados entre 43,55 e 51,94\%. Erros altos também foram encontrados por Pacheco (2013), em que o erro para estimativa do crescimento acumulado em volume para o espaçamento 3,0 × 2,5 m foi de $76,95 \%$.

Em geral, as piores estimativas foram encontradas para as árvores que sofreram danos do tipo anelamento, o qual é considerado mais grave, afetando mais as características de crescimento das árvores, tal como crescimento em altura pela quebra da ponteira das árvores. Algumas variáveis apresentaram erros altos nas estimativas e isto se deve à grande variabilidade dessas variáveis em cada idade, principalmente após os danos causados pelos macacos, que modifica o padrão de crescimento esperado da árvore.

Podem-se observar na Figura 2 as curvas de crescimento e produção em DAP, área transversal, altura e volume de árvores danificadas (aneladas e janeladas) e árvores não danificadas por macacos-prego ao longo dos anos.

A curva de produção em diâmetro para as árvores aneladas, até aproximadamente 15 anos, encontra-se acima das curvas de produção das árvores janeladas e não danificadas. As árvores janeladas apresentaram crescimento inicial até aproximadamente seis anos superior às árvores não danificadas. A partir de seis anos as curvas das árvores janeladas e não danificadas apresentam uma produção semelhante até aproximadamente 15 anos, quando então, a curva de produção das árvores janeladas se torna um pouco superior às árvores não danificadas. As três curvas de produção chegaram aos 18 anos com praticamente os mesmos valores médios de produções em diâmetro $(30,9 \mathrm{~cm}$ para árvores não danificadas, $32,3 \mathrm{~cm}$ para árvores janeladas e $31,8 \mathrm{~cm}$ para árvores aneladas), com a curva de produção em diâmetro das árvores janeladas pouco superior à das árvores aneladas e não danificadas.

O ponto de máximo incremento médio anual IMA em diâmetro para as árvores do povoamento danificado (aneladas e janeladas) ocorreu, respectivamente, aos sete e oito anos, enquanto as árvores do povoamento não danificado ocorreram mais tarde, aos nove anos e meio.

$\mathrm{Na}$ idade de 18 anos, as árvores janeladas apresentaram maior incremento médio anual (IMA) em diâmetro com casca $\left(1,77 \mathrm{~cm}^{-a n o^{-1}}\right)$, seguidas das aneladas e não danificadas, com respectivamente, 1,75 e 1,71 cm.ano-1. Nota-se que aos 18 anos, os valores de produções acumuladas em diâmetro são bastante próximos. 


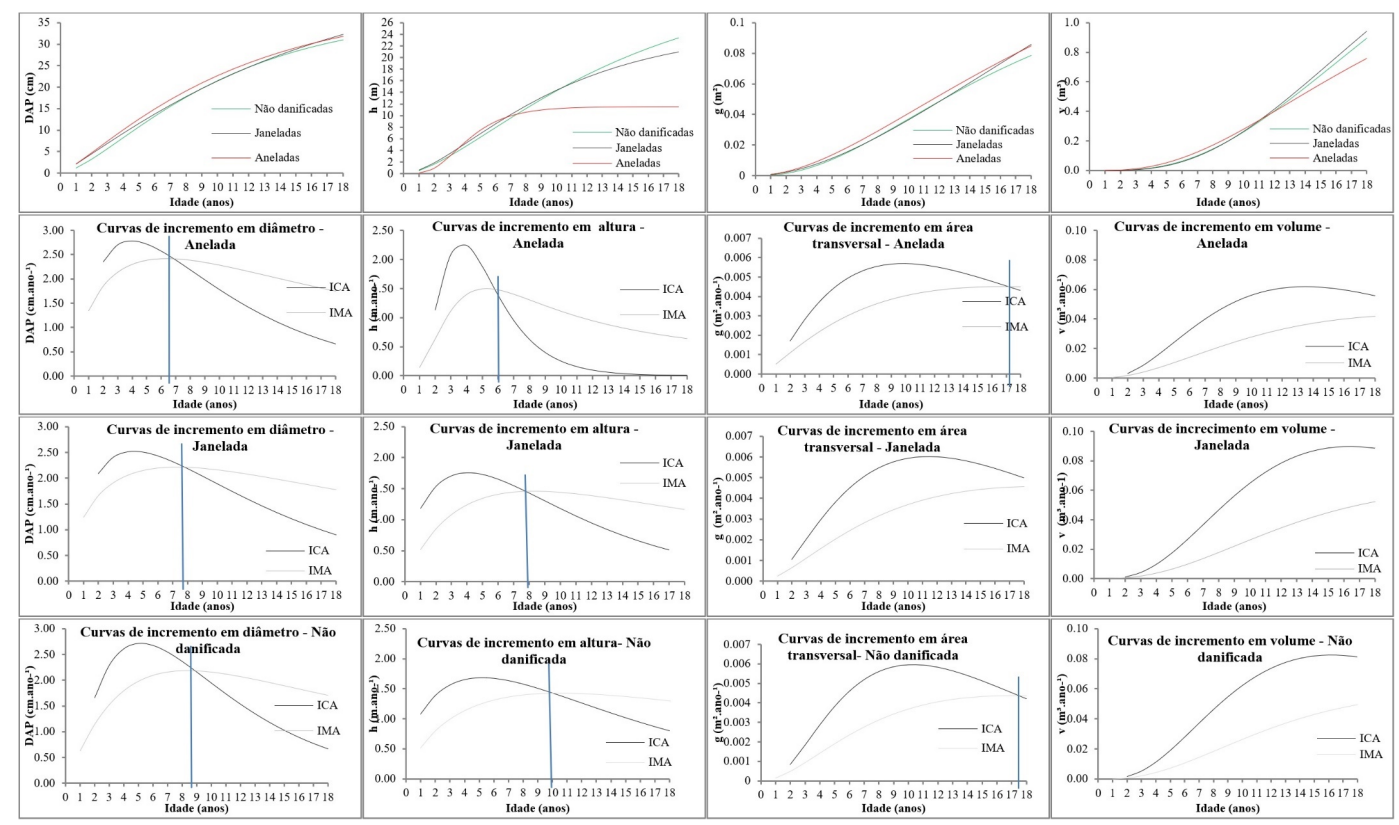

Figura 2. Curvas de crescimento e produção em DAP, área transversal, altura e volume de árvores danificadas (aneladas e janeladas) e árvores não danificadas por macacos-prego.

As curvas de produção apresentadas na Figura 2 mostram o impacto no crescimento em altura para as árvores que sofreram danos do tipo anelamento. A partir de dez anos a curva de produção se estabilizou mantendo-se a uma altura média de aproximadamente de $11,5 \mathrm{~m}$, enquanto árvores não danificadas e janeladas atingiram, aos 18 anos, respectivamente, cerca de 23 e $20 \mathrm{~m}$. Essa redução do crescimento em altura para árvores aneladas foi de, aproximadamente, $50 \%$ do potencial de crescimento que essas árvores poderiam atingir aos 18 anos, comparadas às árvores não danificadas.

As curvas de incremento corrente anual (ICA) e incremento médio anual (IMA) da altura cruzaram, aproximadamente, aos seis, oito e dez anos para as árvores aneladas, janeladas e não danificadas, respectivamente.

O incremento médio anual em altura, aos 18 anos, foi maior para as árvores não danificadas $\left(1,25 \mathrm{~m}\right.$.ano-1), seguido das árvores janeladas $\left(1,16 \mathrm{~m} \cdot \mathrm{ano}^{-1}\right) \mathrm{e}$, por último, as árvores que foram aneladas pelos macacos-prego e tiveram a ponteira seca, estacionando o crescimento do fuste principal $\left(\mathrm{IMA}_{h}=0,64 \mathrm{~m} \cdot \mathrm{ano}^{-1}\right)$. Neste caso, no cálculo do incremento médio anual em altura não foram utilizadas as alturas das brotações que ocorrem quando a árvore anelada não morreu após a queda da ponteira que secou.

As curvas de crescimento em área transversal para as árvores não danificadas, janeladas e aneladas por macacos-prego estão apresentadas na Figura 2. A curva de produção em área transversal para as árvores aneladas manteve-se acima das curvas de produção das árvores janeladas e não danificadas até aproximadamente 15,5 anos. Deste ponto em diante a curva se iguala à curva das árvores janeladas. As curvas de produção em área transversal para árvores janeladas e não danificadas estão sobrepostas até aproximadamente 14 anos, após esta idade até os 18 anos, o crescimento das árvores janeladas se torna superior ao crescimento acumulado das árvores não danificadas.

As curvas de incremento em área transversal para as árvores aneladas e não danificadas cruzaram-se após os dezessete anos. Para as árvores janeladas, as curvas ainda não se cruzaram, ou seja, não atingiram o máximo incremento médio (IMAg), indicando que a área transversal ainda está aumentando para essas situações (Figura 2).

Pode-se observar como os danos do macaco-prego afetaram a produção do volume total com casca ao longo dos anos (Figura 2). Nota-se que, até os dez anos, a produção em volume das árvores aneladas era superior ao das árvores janeladas e não danificadas. A partir dessa idade a produção em volume das árvores janeladas e não danificadas é superior, aumentando essa diferença com o avanço da idade. Aos 18 anos, há uma 
produção maior de volume nas árvores janeladas $\left(0,92697 \mathrm{~m}^{3}\right)$, seguido das não danificadas $\left(0,88944 \mathrm{~m}^{3}\right)$ e aneladas $\left(0,76170 \mathrm{~m}^{3}\right)$.

O volume superior das árvores que sofreram danos do tipo janelamento em relação às árvores sem danos pode ser reflexo do processo de cicatrização que acontece nas árvores após a abertura de fissuras, como é o caso das janelas realizadas pelos macacos-prego. As árvores possuem mecanismos de defesa e, segundo Ferreira \& Milani (2002), uma planta lenhosa afetada superficialmente, por uma injúria (biótica ou abiótica) ou por uma lesão causada por um patógeno na casca, sem atingir o câmbio, é capaz de iniciar um processo de defesa ou barreira fisiológica e morfológica impermeabilizante na casca.

Assim, quando uma árvore é ferida, como no caso da abertura de fissuras pelos macacos-prego, doenças e fungos apodrecedores podem infectar o local e causar danos para se proteger contra a expansão, sendo capazes de selar ou compartimentalizar a área ferida. Dessa forma, as árvores não conseguem substituir os tecidos lesionados, mas sim, os compartimentalizam "gerando" um novo tecido que exerce a função do tecido danificado. Essa forma de defesa em nível de casca é facilmente perceptível, sendo tecnicamente denominado mecanismo de defesa de compartimentalização do lenho (Shigo, 1972 apud Ferreira \& Milani, 2002) e pela formação de periderme necrofilática (Mullick, 1977 apud Ferreira \& Milani, 2002). Desta forma, essa compartimentalização ocorre devido a ação conjunta de várias barreiras, que têm a função de atrasar o ritmo de inativação e apodrecimento do lenho anterior (LA), para dar tempo do novo xilema (lenho posterior - LP) ser produzido (Ferreira \& Milani, 2002).

A primeira barreira (B1) a ser formada pode ser encontrada no xilema com a formação de obstrução nos vasos (tiloses) acima e abaixo do local da agressão para restringir a invasão microbiana no sentido vertical. A segunda barreira (B2) é visualizada no xilema mais fino e resistente, constituída pelo lenho outonal (Figura 3). A terceira barreira é constituída pela compartimentalização causada pelas células do raio (B3). Quando o lenho é submetido a uma agressão (biótica ou abiótica), o câmbio justaposto à área afetada, produz uma nova porção de xilema anatômica e quimicamente distinta do xilema anterior, constituindo a barreira 4 (B4), que é a mais resistente à colonização microbiana (Ferreira \& Milani, 2002).

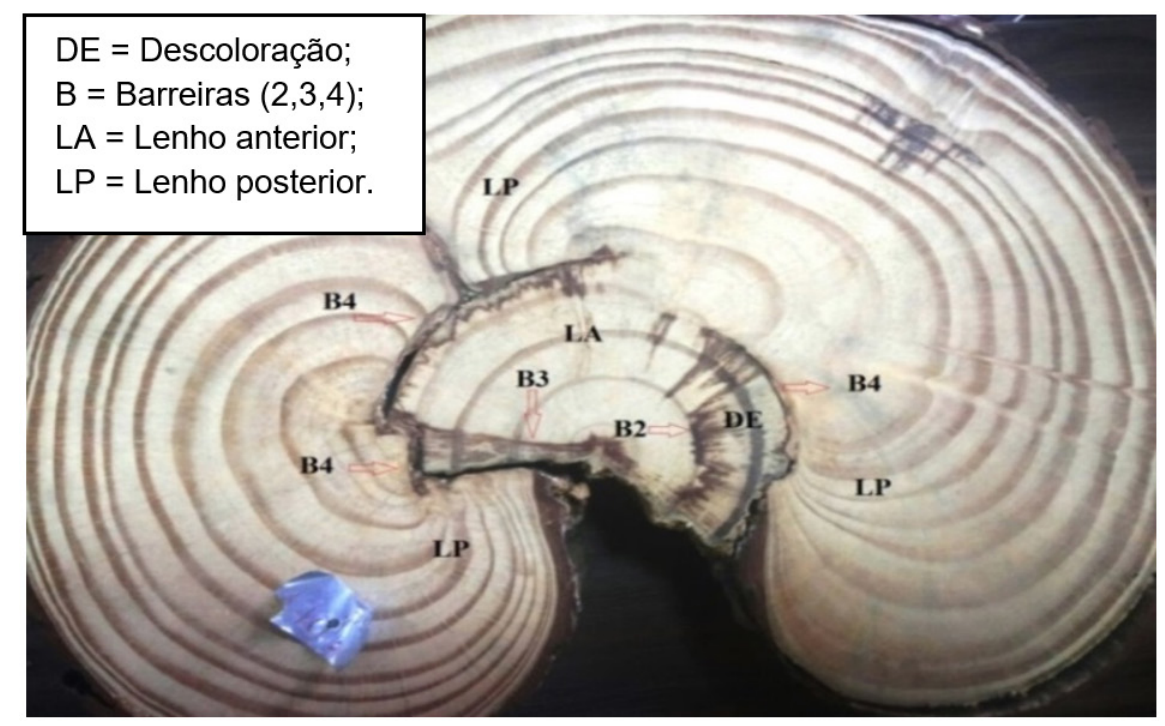

Figura 3. Sistema de compartimentalização do lenho em árvores após danos de macacos-prego em Pinus taeda. Fonte: Retslaff (2018).

Esse processo de compartimentalização e formação de um novo lenho fez com que as árvores, na área lesionada, tenham um aumento em seu diâmetro. Considerando o fato de que, em uma única árvore foi possível encontrar mais de uma janela (até 5 janelas), com comprimento médio de 2,4 m, os mecanismos de defesa das árvores alteraram os diâmetros 
ao longo do fuste e, por consequência, alteram o volume dessas árvores danificadas, porém não se trata de um volume inteiramente útil.

Esse aumento de diâmetro também pode ser observado nos gráficos de perfil do fuste ao longo dos anos para as árvores que foram janeladas conforme Figura 4. Nessa figura são apresentados três perfis, sendo os dois primeiros referentes às árvores com danos do tipo janelamento, e o terceiro trata-se do perfil de uma árvore normal sem danos, para critério de comparação.
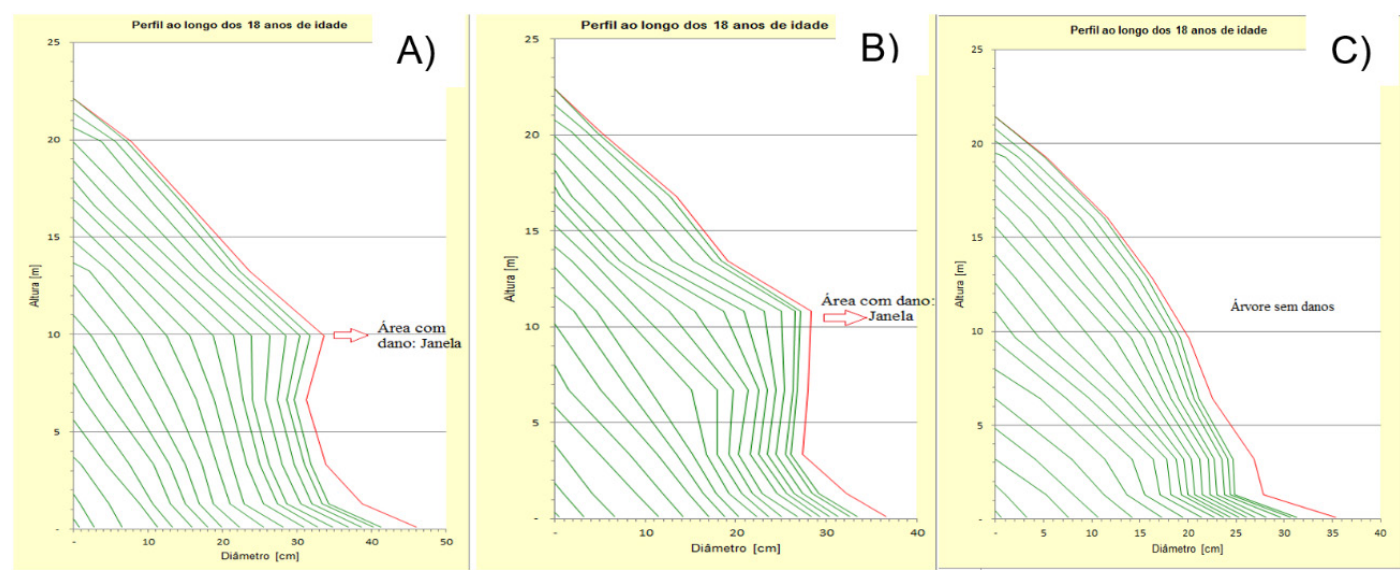

Figura 4. Perfil do fuste de árvores com danos do tipo janelamento (A e B) e perfil de uma árvore sem danos (C).

Também deve-se levar em consideração que nas seções danificadas, as seções se tornam mais irregulares e excêntricas (não se assemelhando a nenhuma figura geométrica conhecida). Assim, quanto mais a área transversal da seção danificada se afastar da forma do círculo, maiores serão os erros na obtenção dessa área transversal, acarretando em uma superestimativa dessa área e consequentemente no volume.

Para algumas árvores, a porção danificada não cicatrizou completamente, levando a seção do dano à formação de um espaço vazio em parte da seção (Figura 3). No entanto, a área transversal da seção é calculada inserindo esse espaço vazio, resultando em um volume sólido maior que o real, mas variando em função da extensão do dano.

As curvas de incremento para volume para os três grupos estudados (aneladas, janeladas e não danificadas) não atingiram o ponto máximo ou o ponto de culminação das curvas de ICA e IMA (Figura 2). Esse resultado é compatível com a tendência normal observada para florestas plantadas em que a variável volume culmina depois das demais variáveis da árvore (Scolforo, 1998; Pacheco, 2013; Machado et al., 2015). Acrescente-se ainda, que dois desbastes realizados (aos 10 e 15 anos) remanescendo 311 árvores.ha-1 no talhão danificado e 352 árvores.ha-1 no talhão não danificado, podem ter retardado a culminação dessas curvas.

O incremento médio anual (IMA) estimado em volume para as árvores com danos do tipo janelamento, anelamento e não danificadas foi de 0,051 $\mathrm{m}^{3}$.ano-1, $0,041 \mathrm{~m}^{3}$.ano-1 e 0,049 $\mathrm{m}^{3}$.ano-1, respectivamente, aos 18 anos de idade. Para os três grupos de árvores estudados as curvas ainda não se cruzaram, ou seja, não atingiram o máximo incremento médio anual, indicando que o volume ainda está aumentando (Figura 2).

O teste de igualdade de parâmetros e identidade de modelos de regressão não-lineares, proposto por Regazzi (2003), foi aplicado para avaliar a influência dos danos de macacos-prego na produção das variáveis estudadas para Pinus taeda. Os coeficientes do modelo de Chapman-Richards foram testados separadamente para o ajuste de cada variável (DAP, g, h e v) para os três grupos de árvores analisados (não danificadas, aneladas e janeladas), porém, apenas para a variável altura (h) os coeficientes " $A$ ", " $b$ " e " $C$ ", foram diferenciados estatisticamente pelo teste de igualdade de parâmetros (Tabela 2).

O teste identificou que, para as variáveis DAP, $h$ e $v$, as funções de crescimento devem ser ajustadas para cada grupo, pois a hipótese $\mathrm{H}_{04}$ foi rejeitada. Concluindo-se que as curvas de produção para essas variáveis não são iguais e que os danos dos macacos-prego influenciam no crescimento das árvores de Pinus taeda (Tabela 2). 
Tabela 2. Teste de igualdade de parâmetros e identidade de modelos de regressão não-linear proposto por Regazzi (2003), aplicado para o modelo de Chapman-Richards ajustado para o diâmetro (DAP), área transversal, altura e volume com casca para cada grupo de árvores estudadas (não danificadas, janeladas e aneladas).

\begin{tabular}{|c|c|c|c|c|c|c|c|c|c|c|c|}
\hline & \multicolumn{5}{|c|}{ Diâmetro (DAP) } & & \multicolumn{5}{|c|}{ Área transversal (g) } \\
\hline & \multirow{2}{*}{ HI } & $\mathrm{H}_{01}$ & $\mathrm{H}_{02}$ & $\mathrm{H}_{03}$ & $\mathrm{H}_{04}$ & & \multirow{2}{*}{ HI } & $\mathrm{H}_{01}$ & $\mathrm{H}_{02}$ & $\mathrm{H}_{03}$ & $\mathrm{H}_{04}$ \\
\hline & & A & B & C & $A, b, c$ & & & A & B & C & $A, b, c$ \\
\hline $\mathrm{A} 1$ & 35,247 & 36,297 & 36,226 & 36,735 & 36,317 & $\mathrm{~A} 1$ & 0,1206 & 0,1289 & 0,1298 & 0,1333 & 0,1297 \\
\hline $\mathrm{A} 2$ & 40,06 & 36,297 & 36,706 & 37,413 & 0,1392 & $\mathrm{~A} 2$ & 0,1502 & 0,1289 & 0,1359 & 0,1451 & 0,0984 \\
\hline A3 & 36,015 & 36,297 & 36,074 & 34,95 & 1,7661 & A3 & 0,1302 & 0,1289 & 0,1209 & 0,1125 & 2,6474 \\
\hline b1 & 0,1514 & 0,14 & 0,1398 & 0,1334 & 36,317 & b1 & 0,1115 & 0,1013 & 0,1 & 0,0958 & 0,1297 \\
\hline b2 & 0,1107 & 0,142 & 0,1398 & 0,1339 & 0,1392 & b2 & 0,0872 & 0,107 & 0,1 & 0,0915 & 0,0984 \\
\hline b3 & 0,1406 & 0,137 & 0,1398 & 0,1559 & 1,7661 & b3 & 0,0893 & 0,0906 & 0,1 & 0,1134 & 2,6474 \\
\hline c1 & 1,9858 & 1,864 & 1,8553 & 1,7738 & 36,317 & $\mathrm{c} 1$ & 2,9887 & 2,7945 & 2,7618 & 2,6709 & 0,1297 \\
\hline c2 & 1,539 & 1,817 & 1,8172 & 1,7738 & 0,1392 & c2 & 2,59 & 2,9519 & 2,9274 & 2,6709 & 0,0984 \\
\hline c3 & 1,6174 & 1,591 & 1,6108 & 1,7738 & 1,7661 & c3 & 2,2633 & 2,2817 & 2,4253 & 2,6709 & 2,6474 \\
\hline SQ res & 20239,4 & 20270,1 & 20265,1 & 20277,4 & 20691 & SQ res & 0,3195 & 0,3197 & 0,31965 & 0,3197 & 0,3213 \\
\hline $\mathbf{N}$ & 1251 & 1251 & 1251 & 1251 & 1251 & $\mathbf{N}$ & 1229 & 1229 & 1229 & 1229 & 1229 \\
\hline$\chi^{2}$ cal & & $2,214^{\mathrm{ns}}$ & $1,921^{\mathrm{ns}}$ & $2,269^{\text {ns }}$ & $27,606 *$ & $\chi^{2}$ cal & & $0,5421^{\text {ns }}$ & $0,7958^{\text {ns }}$ & $0,3537^{\text {ns }}$ & 6,7732 ns \\
\hline GI & 9 & 7 & 7 & 7 & 3 & GI & 9 & 7 & 7 & 7 & 3 \\
\hline \multirow[t]{4}{*}{$\chi^{2}$ tab } & & 5,991 & 5,991 & 5,991 & 12,591 & $\chi^{2}$ tab & & 5,991 & 5,991 & 5,991 & 12,591 \\
\hline & \multicolumn{5}{|c|}{ Altura (h) } & & \multicolumn{5}{|c|}{ Volume $\left(\mathrm{m}^{3}\right)$} \\
\hline & $\mathrm{HI}$ & $\mathbf{H}_{01}$ & $\mathrm{H}_{02}$ & $\mathrm{H}_{03}$ & $\mathrm{H}_{04}$ & & \multirow{2}{*}{ HI } & $\mathrm{H}_{01}$ & $\mathrm{H}_{02}$ & $\mathrm{H}_{03}$ & $\mathrm{H}_{04}$ \\
\hline & & A & B & c & $A, b, c$ & & & A & b & C & $A, b, c$ \\
\hline $\mathrm{A} 1$ & 32,6313 & 15,0994 & 28,9059 & 30,6795 & 23,0297 & $\mathrm{~A} 1$ & 2,2184 & 1,945 & 2,0313 & 2,1799 & 1,9616 \\
\hline $\mathrm{A} 2$ & 24,4269 & 15,0994 & 25,4747 & 24,4092 & 0,1284 & $\mathrm{~A} 2$ & 1,7959 & 1,945 & 2,1379 & 2,3178 & 0,092 \\
\hline A3 & 11,5308 & 15,0994 & 13,5256 & 11,8912 & 1,6703 & $\mathrm{~A} 3$ & 1,4903 & 1,945 & 1,493 & 1,2905 & 3,9041 \\
\hline b1 & 0,097 & 0,4787 & 0,126 & 0,111 & 23,0297 & b1 & 0,088 & 0,0998 & 0,0959 & 0,0895 & 1,9616 \\
\hline b2 & 0,1413 & 0,4732 & 0,126 & 0,1416 & 0,1284 & b2 & 0,1148 & 0,1058 & 0,0959 & 0,088 & 0,092 \\
\hline b3 & 0,4833 & $4,17 E+13$ & 0,126 & 0,3063 & 1,6703 & b3 & 0,0961 & 0,0725 & 0,0959 & 0,1149 & 3,9041 \\
\hline c1 & 1,7383 & 9,0233 & 2,0743 & 1,9035 & 23,0297 & c1 & 3,9667 & 4,3371 & 4,2156 & 4,0149 & 1,9616 \\
\hline c2 & 1,8999 & 7,7981 & 1,7365 & 1,9035 & 0,1284 & c2 & 4,9958 & 4,6532 & 4,2942 & 4,0149 & 0,092 \\
\hline c3 & 4,602 & $6,99 E+09$ & 0,791 & 1,9035 & 1,6703 & c3 & 3,4729 & 2,9425 & 3,4679 & 4,0149 & 3,9041 \\
\hline SQ res & 3554,213 & 27362,7 & 3852,8 & 3623,77 & 13043,78 & SQ res & 34,091 & 34,116 & 34,098 & 34,107 & 34,99 \\
\hline $\mathbf{N}$ & 1255 & 1255 & 1255 & 1255 & 1255 & $\mathbf{N}$ & 1249 & 1249 & 1249 & 1249 & 1249 \\
\hline$\chi^{2}$ cal & & $2561,514^{*}$ & $101,236 *$ & $24,323 *$ & $1631,723^{*}$ & $\chi^{2}$ cal & & $0,9155^{\mathrm{ns}}$ & $0,260^{\text {ns }}$ & $0,597^{\text {ns }}$ & $32,516 *$ \\
\hline GI & 9 & 7 & 7 & 7 & 3 & GI & 9 & 7 & 7 & 7 & 3 \\
\hline$\chi^{2}$ tab & & 5,991 & 5,991 & 5,991 & 12,591 & $\chi^{2}$ tab & & 5,991 & 5,991 & 5,991 & 12,591 \\
\hline
\end{tabular}

Em que: ns = não significativo ao nível de $5 \%$ de probabilidade; *significativo ao nível de $5 \%$ de probabilidade; SQres = soma de quadrados de resíduos; $\mathrm{n}$ = número de observações; $\chi^{2}$ calc = Qui-quadrado calculado; $\mathrm{GL}=$ graus de liberdade; $\chi^{2}$ tab = Qui-quadrado tabelado. OBS: células marcadas são referentes aos coeficientes que foram estimados como sendo iguais para os três grupos de dados; $\mathrm{HI}_{1} \mathrm{H}_{01}, \mathrm{H}_{02}, \mathrm{H}_{03}$ e $\mathrm{H}_{0}=$ hipóteses do teste (descritas na metodologia); A, b, c = parâmetros.

O povoamento estudado sofreu danos ao longo de sua vida, porém, como descrito por Liebsch et al. (2015), quanto mais jovem for o povoamento no momento da ocorrência dos danos, maiores serão os prejuízos econômicos, pois acabam danificando as árvores entre cinco e sete metros de altura, atingindo partes mais nobres do fuste que possuem alto valor comercial.

Nesse contexto, buscou-se identificar a idade em que ocorreram os primeiros danos. Para tanto, foi utilizado os dados da análise de tronco e avaliou, se os gráficos de perfil do fuste gerados e a idade das brotações, que também auxiliaram na identificação dessas idades. Nas brotações, a idade foi verificada retirando-se um disco da base da maior ramificação presente em cada árvore.

Constatou-se que os danos às árvores do talhão tiveram início quando o povoamento tinha entre cinco e seis anos de idade (Figura 5A), comprovado por meio das idades das brotações, as quais tinham entre 12 e 13 anos (Figura 5B). Porém, 52\% das árvores 
amostradas que foram aneladas e janeladas apresentaram seus primeiros danos entre sete e oito anos (Figura 5C).

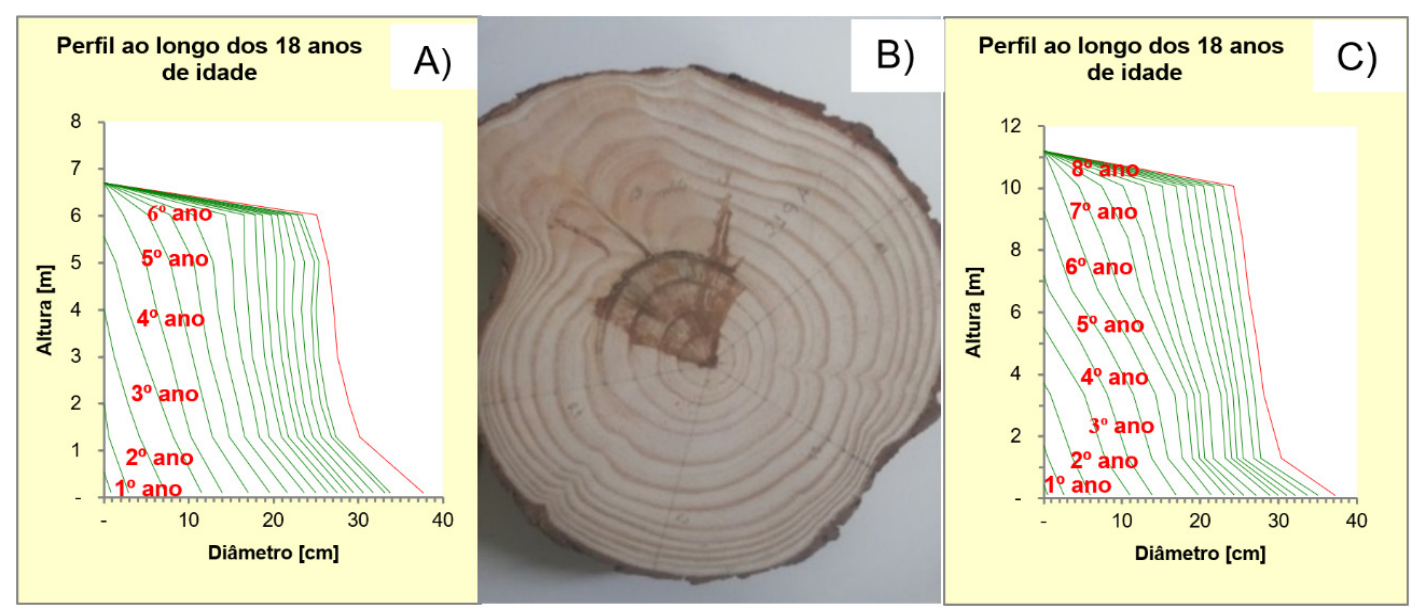

Figura 5. Perfil do fuste (A) indicando a ocorrência do primeiro dano do macaco-prego (5-6 anos) e disco da base da ramificação apontando para 12-13 anos (B). Perfil do fuste, anelado entre 7 e 8 anos de idade (C).

\section{CONCLUSÃO}

- A análise de tronco mostrou ser uma excelente técnica para identificar as idades de ocorrência dos danos pelos macacos-prego.

- A altura foi a variável mais afetada pelos danos de macacos-prego, reduzindo em cerca de 50\% seu potencial de crescimento para as árvores aneladas e, como consequência, afetou também a produção volumétrica.

- $\quad$ As árvores janeladas produziram mais volume que as árvores não danificadas e isto se deve ao processo de compartimentalização das fissuras abertas pelos macacos-prego e também pelo tamanho dessas fissuras, que fizeram com que os diâmetros ao longo do fuste aumentassem.

- $\quad$ O teste de Regazzi (2003) apontou diferença estatística entre as curvas de crescimento individuais, não sendo, portanto, possível o uso de uma única equação para os três grupos de árvores estudados.

- Os primeiros danos ao povoamento de Pinus taeda estudado ocorreram entre cinco e seis anos, mas o maior porcentual de danos se deu entre sete e oito anos de idade.

\section{REFERÊNCIAS BIBLIOGRÁFICAS}

Alfaro, J. W. L., Boubli, J. P., Olson, L. E., Di Fiore, A., Wilson, B., Gutiérrez-Espeleta, G. A., Chiou, K. L., Schulte, M., Neitzel, S., Ross, V., Schwochow, D., Nguyen, M. T. T., Farias, I., Janson, C. H., \& Alfaro, M. E. (2012). Explosive Pleistocene range expansion leads to widespread Amazonian sympatry between robust and gracile capuchin monkeys. Journal of Biogeography, 39(2), 272-288. http://dx.doi.org/10.1111/j.1365-2699.2011.02609.x.

Carvalho, D. R. J. (2007). Predação em Pinus spp. por Cebus nigritus (Goldfuss, 1809) (Primates; Cebidae) na região nordeste do Paraná Brasil (Dissertação de mestrado). Universidade Federal do Paraná, Curitiba.

Ferreira, F. A., \& Milani, D. (2002). Diagnose visual e controle das doenças abióticas e bióticas do eucalipto no Brasil (pp. 98). Mogi Guaçu: International Paper.

Indústria Brasileira de Árvores - IBÁ. (2017). Relatório 2016. Brasília: IBÁ. Recuperado em 4 de janeiro de 2019, de https://iba.org/datafiles/publicacoes/pdf/iba-relatorioanual2017.pdf

Jungles, D. R. C., \& Vidolin, G. P. (2009). Delineamentos para o manejo do macaco-prego Cebus nigritus. (1 ${ }^{\text {a }}$ Ed. Vol. 1, pp. 13-32) Curitiba: IAP.

Liebsch, D., Mikich, S. B., Oliveira, E. B., \& Moreira, J. M. M. A. P. (2015). Descascamento de Pinus taeda por macacos-prego (Sapajus nigritus): tipos e intensidades de danos e seus impactos sobre o crescimento das árvores. Scientia Forestalis, 43, 37-49. 
Liebsch, D., Moreira, J. M. M. A. P., Oliveira, E. B., \& Mikich, S. B. (2018). Impacto do descascamento de árvores de Pinus taeda L. por macacos-prego na produção e receita em plantios com desbastes. Biofix Scientific Journal, 3(1), 48-55. http://dx.doi.org/10.5380/biofix.v3i1.56574.

Machado, S. A., Souza, R. F., Aparecido, L. M. T., Ribeiro, A., \& Czelusniak, B. H. (2015). Evolução das variáveis dendrométricas da bracatinga por classe de sítio. Cerne, 21(2), 199-207. http://dx.doi.org/10.1590/01047760201521021222.

Mikich, S. B., Liebsch, D., Almeida, A., Oliveira, E., \& Mauro, J. (2015). O programa macaco-prego da EMBRAPA florestas: avanços e perspectivas. In Anais do $5^{\circ}$ Congresso Florestal Paranaense (pp. 1-17). Porto Alegre: AGEFLOR.

Oliveira, E. B., Moreira, J. M. A., Liebsch, D., \& Mikich, S. B. (2015). Ferramenta computacional para estimar perdas na produção de madeira de Pinus taeda em plantações danificadas por macaco-prego (Sapajus nigritus). Colombo: Embrapa Florestas.

Pacheco, J. M. (2013). Influência do espaçamento no crescimento de Pinus taeda L. na região Centro-Sul do Paraná (Dissertação de mestrado). Universidade Estadual do Centro-Oeste, Irati.

Pavelski, J. A. (2016). Quantificação das perdas ocasionadas por ataque de macaco-prego em reflorestamentos de Pinus taeda (Monografia). Universidade Federal do Paraná, Curitiba.

Regazzi, A. J. (2003). Teste para verificar a igualdade de parâmetros e a identidade de modelos de regressão não linear. Ceres, 50, 9-26.

Retslaff, M. S. F. (2018). Crescimento e produção em povoamentos de Pinus taeda L. danificados por macacos-prego e seus impactos econômicos. (Dissertação de Mestrado). Universidade Estadual do Centro Oeste, Irati.

Rocha, V. J. (2000). Macaco-prego, como controlar esta nova praga florestal? Floresta, 30(1-2), 95-99. http://dx.doi.org/10.5380/rf.v30i12.2329.

Scolforo, J. R. S. (1998). Biometria florestal: modelagem do crescimento e da produção de florestas plantadas e nativas (441 p.). Lavras: UFLA/FAEPE.

Wolff II, I. N. (2012). Modelagem do crescimento e da produção de Pinus taeda L (Dissertação de mestrado). Universidade Estadual do Centro-Oeste, Irati.

Contribuição dos Autores: FMSR: Conceptualização, Curadoria de dados, Análise formal, Investigação, Metodologia, Administração do projeto, Supervisão, Visualização, Redação - rascunho original, Redação revisão e edição; AFF: Conceptualização, Análise formal, Metodologia, Administração do projeto, Supervisão, Visualização, Redação revisão e edição; FASR: Conceptualização, Análise formal, Metodologia, Administração do projeto, Supervisão, Visualização, Redação revisão e edição; JRAM: Análise formal, Metodologia, Administração do projeto, Supervisão, Visualização, Redação - rascunho original, Redação revisão e edição. 